\title{
Purification and Chemical Properties of Anti-complementary Polysaccharide from the Leaves of Artemisia princeps
}

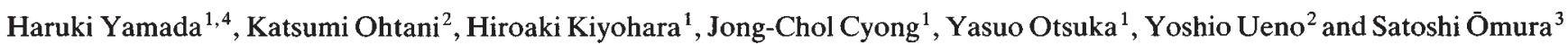

Received: August 28, 1984; Accepted: December 25, 1984

\begin{abstract}
The polysaccharide fraction from the leaves of Artemisia princeps Pamp (Japanese name = Gaiyō) showed a potent anti-complementary activity. Two major active polysaccharides (AAF-IIb-2 and IIb-3) were purified by ion exchange chromatography on DEAESepharose, affinity chromatography on Ricinus communis-agglutinin conjugated Sepharose and gel filtration on Sephadex G-100 and Sepharose CL-4B. The molecular weights of AAF-IIb-2 and IIb-3 were found to be 139000 and 31000 by the calibration of gel filtration. AAFIIV-2 and IIb- 3 were composed by rhamnose, xylose, arabinose, galactose and glucose in the molar ratios of $2.5: 2.5: 4.3: 3.6: 1.0$ and $1.5: 1.0: 9.4: 7.5: 1.0$, and also contained $59.4 \%$ and $49.0 \%$ galacturonic acid, respectively. AAF-IIb-2 was shown to have the more potent activity than AAF-IIb-3. Anti-complementary activity of AAFIIb-3 was almost similar with that of crude AR-arabinogalactan from the root of Angelica acutiloba Kitagawa, but AAF-IIb-2 and IIb-3 showed more potent activity than anti-complementary $\beta$-glucan, lentinan.
\end{abstract}

\section{Introduction}

It is known that complement system plays an important role in the host defense system, inflammations and allergic reactions. A considerable number of Chinese herbs have been found to be anti-inflammatory (1). These findings present the possibility that these herbs may contain some kinds of regulators of the complement system. Recently, we have found potent anti-complementary activities in the extracts of some Chinese herbs (2), such as in the extract of the leaves of Artemisia princeps Pamp. Artemisiae Argyi Folium, the leaves of $A$. princeps Pamp (Japanese name $=$ Gaiyō) is a well known crude drug clinically used in the treatment of colic pain, vomiting and diarrhea, and irregular bleeding from uterus. The hairs on the undersurface of the leaves of $A$. princeps have also been used as moxa for moxibustion. Previously, we reported that the hot-water extract of moxa contained potent anti-complementary activity, and the activity was observed in the non-dialyzable carbohydrate-rich fraction (3). It was suggested that the active principle may be a kind of polysaccharide molecule.

The present paper describes the purification and chemical characterization of the major anti-complementary polysaccharides from the leaves of $A$. princeps (Pamp) which is the raw material of moxa.

\footnotetext{
${ }^{1}$ Oriental Medicine Research Center of the Kitasato Institute, 5-9-1 Shirokane, Minato-ku, Tokyo 108, Japan

${ }^{2}$ Faculty of Pharmaceutical Sciences, Science University of Tokyo, Shinjuku-ku, Tokyo 162, Japan

3 The Kitasato Institute, Minato-ku, Tokyo 108, Japan

${ }^{4}$ Address for correspondence
}

\section{Materials and Methods}

\section{General methods}

The total carbohydrate and uronic acid contents were determined by the phenol-sulfuric acid method (4) and $m$-hydroxydiphenyl method (5), respectively, using arabinose and galacturonic acid as the respective standards. Protein was assayed by the method of Lowry et al. (6) with bovine serum albumin as the standard. TLC was performed on cellulose-coated plastic sheets (Merck) with EtOAc - pyridine - AcOH $\mathrm{H}_{2} \mathrm{O}(5: 5: 1: 3)$ as the solvent system. Reducing sugars were detected with alkaline silver nitrate (7), and uronic acid with $p$-anisidine hydrochloride (8). GLC (Shimadzu GC-6A) was equipped with a flame ionization detector (FID) and a glass column $(0.3 \times 200 \mathrm{~cm})$ of $3 \%$ ECNSS-M or $1 \%$ Silicon OV-225 on Uniport HP at $180^{\circ} \mathrm{C}$ or $200^{\circ} \mathrm{C}$, respectively. Nitrogen was used as a carrier gas at a flow rate of $60 \mathrm{ml} /$ min. After acid hydrolysis of the polysaccharides, the component aldoses were converted into alditol acetates and analyzed by GLC as described previously (9). Gel filtration was carried out by elution with water or $0.2 \mathrm{M} \mathrm{NaCl}$ on Sephadex G-100 $(3.0 \times 87 \mathrm{~cm})$, Sepharose CL$2 \mathrm{~B}(3 \times 90 \mathrm{~cm})$ or Sepharose CL-4B $(3 \times 92 \mathrm{~cm})$. Con-A Sepharose chromatography was carried out in accordance with the method of Kennedy and Rosevear (10). Electrophoresis on glass-paper (ToyoRoshi GA-100) was carried out in $26 \mathrm{mM}$ borate buffer (pH 9.6) at a constant current of $5 \mathrm{~mA} / \mathrm{cm}$ for $1 \mathrm{~h}$ or in $50 \mathrm{mM}$ acetate buffer (pH 5.6) at a constant current of $5 \mathrm{~mA} / \mathrm{cm}$ for $3 \mathrm{~h}$, and the polysaccharide was detected with the 1-naphthol-sulfuric acid reagent (11).

\section{Preparation of crude polysaccharide from A. princeps Pamp}

The leaves of $A$. princeps Pamp (500 g) were decocted with water (10 l) until this volume was reduced by half, and the residue was then further decocted with water (51) as above. The extracts were combined and lyophilized to give a water-soluble extract (AAF-O, yield $107.8 \mathrm{~g}$ ). $\mathrm{AAF}-\mathrm{O}$ was refluxed with 21 of $\mathrm{MeOH}$ for $1 \mathrm{~h}$ and centrifuged to give a $\mathrm{MeOH}$-insoluble precipitate. The precipitate was dissolved in water and dialyzed against running water for 3 days. After the non-dialyzable portion was centrifuged at 7,500 r.p.m. for $30 \mathrm{~min}$, the supernatant was lyophilized to obtain crude polysaccharide (AAF-1, yield $12.26 \mathrm{~g}$ ). The $\mathrm{MeOH}$-soluble fraction was evaporated to dryness, after which the residue was redissolved in water and lyophilized to obtain AAF-MeOH ext. (yield $36.41 \mathrm{~g}$ ).

\section{Pronase digestion}

AAF-IIb (200 mg) was dissolved in $50 \mathrm{ml}$ of $50 \mathrm{mM}$ Tris- $\mathrm{HCl}$, pH 7.9, containing $10 \mathrm{mM} \mathrm{CaCl}_{2}$, and then $50 \mathrm{mg}$ of Pronase was added. The reaction mixture was incubated at $37^{\circ} \mathrm{C}$ for $48 \mathrm{~h}$ with a small amount of toluene. The reaction was terminated by boiling for $5 \mathrm{~min}$. The mixture was then dialyzed against $\mathrm{H}_{2} \mathrm{O}$ for 2 days, and the non-dialyzable portion was lyophilized to obtain the AAF-IIb Pronase digest.

\section{Periodate oxidation}

AAF-IIb (50 mg) was dissolved in $30 \mathrm{ml}$ of $50 \mathrm{mM}$ acetate buffer, $\mathrm{pH}$ 4.5 , and then $50 \mathrm{mM} \mathrm{NaIO}_{4}(10 \mathrm{ml})$ was added. The reaction mixture was incubated at $4^{\circ} \mathrm{C}$ in the dark for 3 days. Ethylene glycol $(5 \mathrm{ml})$ was added to destroy the excess periodate, and the mixture was dialyzed 
against $\mathrm{H}_{2} \mathrm{O}$ for 2 days. The non-dialyzable solution was concentrated to about $20 \mathrm{ml}$, and $20 \mathrm{mg}$ of $\mathrm{NaBH}_{4}$ was added to the concentrate while being continuously stirred for $12 \mathrm{~h}$ at room temperature. After the neutralization of the reaction mixture with $\mathrm{AcOH}$, the $\mathrm{H}_{3} \mathrm{BO}_{4}$ in the sample was removed by the repeated addition and evaporation of $\mathrm{MeOH}$. Finally, the oxidised AAF-IIb was obtained as the lyophilisate after the dialysis.

\section{Anti-complementary activity}

The anti-complementary activity was measured according to the previously described procedure (12) except distilled water (DIW) was used for dilutions instead of phosphate buffered saline, PBS (pH 7.4).

\section{Materials}

The leaves of Artemisia princeps Pamp (Japanese name $=$ Gaiyō) and the seed of Ricinus communis were purchased from Uchida Wakanyaku Co. Ltd., Tokyo, Japan. Concanavalin-A (Con-A) Sepharose, CNBr-activated Sepharose 4B, Sephadex G-100 and Sepharose CL-4B and CL-2B were obtained from Pharmacia Co. Ltd. Pronase was purchased from Kaken-kagaku Co. Ltd. (Tokyo). Ricinus communis agglutinin (RCA) was purified from the extract of $R$. communis by the method of Adair and Kornfeld (13). RCA-Sepharose was prepared by the coupling of RCA and CNBr-activated Sepharose (14). Lentinan from Lentinas edodes was a gift from Dr. G. Chihara.

\section{Results}

\section{Fractionation of polysaccharides from Artemisia princeps Pamp}

The crude polysaccharide fraction was prepared after the reflux of the lyophilized extract with $\mathrm{MeOH}$. The crude polysaccharide fraction showed potent anti-complementary activity dose dependently but the $\mathrm{MeOH}$-soluble fraction did not show the activity even when concentrated materials were used for the assay. The crude polysaccharide fraction was further separated on the column of DEAE-Sepharose $\left(\mathrm{Cl}^{-}\right)$into unabsorbed fractions (AAF-Ia, b) and absorbed fractions (AAF-IIa, b, c) by the elution with a linear gradient of $\mathrm{NaCl}$ (Fig. 1). Furthermore, the remaining absorbed fraction (AAF-IId) was obtained by the elution with $2 \mathrm{M} \mathrm{NaCl}$ (data not shown). Fig. 2 shows the anti-complementary activity after the incubation of the different concentrations of the polysaccharide fractions with NHS. The anti-complementary activities are shown to be dose dependent. When $1000 \mu \mathrm{g} / \mathrm{ml}$ of IIa was incubated with an equal volume of NHS about $90 \%$ of the $\mathrm{TCH}_{50}$ was reduced. The order of the activities of these fractions was IIa $>$ IIb $>$ Ia $>$ IIc $>$ IId $>$ Ib. The chemical properties of these subfractions

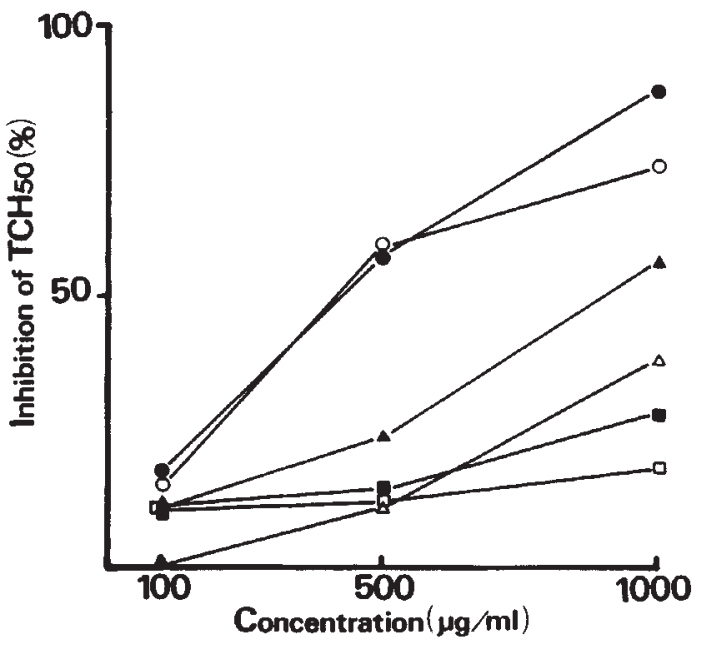

Fig. 2. Anti-complementary activity of polysaccharide fractions from Fig. 1 on DEAE-Sepharose CL-6B.

$\triangle \mathrm{AAF}-\mathrm{Ia}, \square$ AAF-Ib, AAF-IIa, O AAF-IIb, $\triangle$ AAF-Ilc, $\square$ AAFIId.

are summarized in Table $\mathrm{I}$. The most active fraction, IIa, contained arabinose, galactose, mannose, glucose and uronic acid. The major polysaccharide fraction, IIb, contained arabinose, galactose, and xylose as the major neutral sugar, and a large amount of uronic acid which was estimated to be galacturonic acid by TLC of the acid hydrolyzates.

Table I. Chemical Properties of Polysaccharide Fraction from A. Princeps

\begin{tabular}{lrrrrrr}
\hline (\%) & Ia & Ib & IIa & IIb & IIc & IId \\
\hline Total hexose & 45.8 & 44.6 & $117.3^{\mathrm{a}}$ & 70.5 & 38.5 & 34.3 \\
Total uronic acid & 0.4 & 0.8 & 11.0 & 71.6 & 4.5 & 8.8 \\
Total protein & 1.0 & 6.2 & 6.2 & 8.6 & 31.7 & 79.5 \\
\hline (Component sugars) & & \multicolumn{5}{c}{ (Molar ratios) } \\
Rhamnose & - & - & 0.7 & 8.1 & - & 7.3 \\
Arabinose & 54.1 & 30.5 & 38.8 & 36.4 & 78.0 & 30.7 \\
Xylose & 2.3 & 2.5 & 3.9 & 14.8 & 1.7 & 2.5 \\
Mannose & 12.5 & 19.5 & 16.2 & 6.5 & 3.2 & 4.1 \\
Galactose & 11.9 & 14.4 & 26.5 & 29.3 & 11.3 & 30.1 \\
Glucose & 5.7 & 33.2 & 13.8 & 4.9 & 5.7 & 25.3 \\
\hline
\end{tabular}

${ }^{a}$ High value was given by phenol-sulfuric acid assay using arabinose as the standard because hexose is more sensitive than arabinose in this assay.

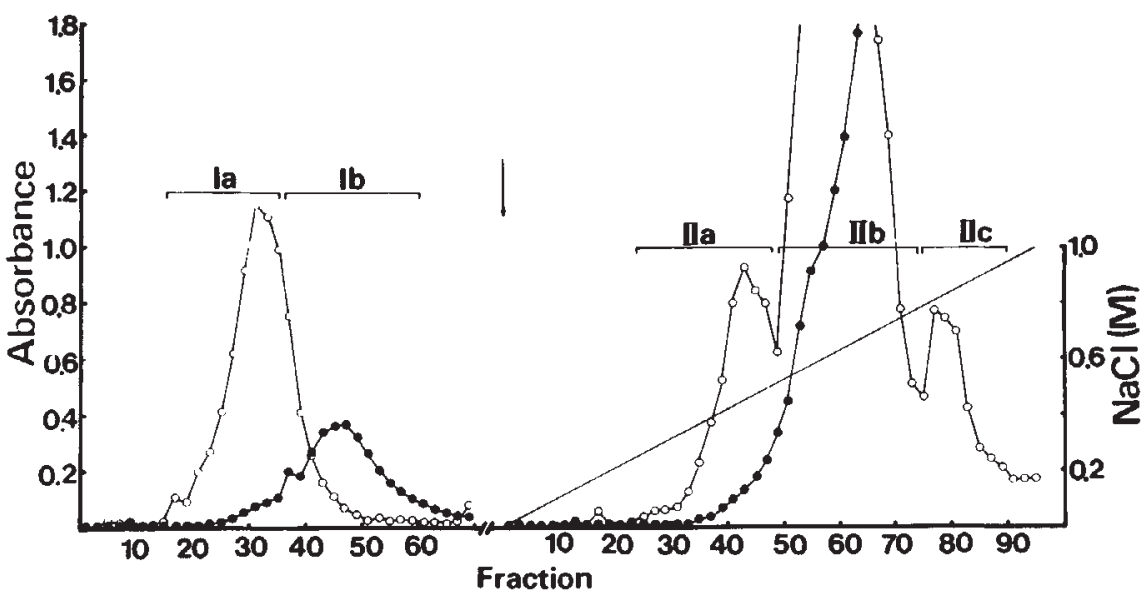

Fig. 1. DEAE Sepharose CL-6B ( $\mathrm{Cl}^{-}$form) chromatography of the crude polysaccharide (AAF-1) from $A$. princeps Pamp. AAF-1 was dissolved in distilled $\mathrm{H}_{2} \mathrm{O}$. Linear gradient elution was carried out with $0-1 \mathrm{M} \mathrm{NaCl}(-)$. Column size $(3 \times 35 \mathrm{~cm})$. Carbohydrate, 490 $\mathrm{nm}(\mathrm{O})$; protein, $280 \mathrm{~nm}(\bullet)$. 
This fraction was used for further purification. The anti-complementary activity of the deproteinized IIb did not change significantly, compared with that of IIb, but its activity was found to decrease with the periodate oxidation of IIb (Fig. 3). The yield of AAF-IIl was $25.5 \%$ from the crude polysaccharide fraction.

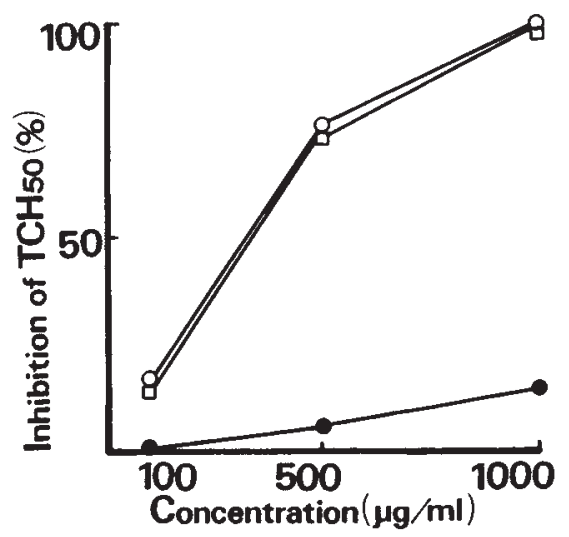

Fig. 3. Lability of polysaccharide anti-complementary activity to periodate and Pronase treatment.

AAF-IIb, $\square$ AAF-IIb Pronase digest, $\mathrm{NaIO}_{4}$ oxidized AAF-IIb.

Purification of the anti-complementary polysaccharide, AAFIIb

AAF-IIb was found to contain a remarkable amount of galactose and was further purified by affinity chromatography on RCA-Sepharose. The RCA unbound fractions showed higher activity than the bound fraction (data not shown). The RCA unbound fraction (yield; $92.5 \%$ from AAF-IIb) was further fractionated by Con-A Sepharose because the RCA-unbound fraction was found to contain mannose. However the amount of the Con-A bound fraction obtained by this second affinity chromatography was insignificant. The RCA-unbound fraction was further purified by gel filtration on Sephadex G-100 as shown in Fig. 4. The anti-complementary active polysaccharide fraction was eluted in the void volume. The fraction eluted in the void volume (yield; $13 \%$ from RCA-unbound fraction) yielded galactose, xylose, rhamnose, arabinose, galacturonic acid and a trace of mannose on hydrolysis whereas the fraction of smallest molecular weight contained a large amount of galac-

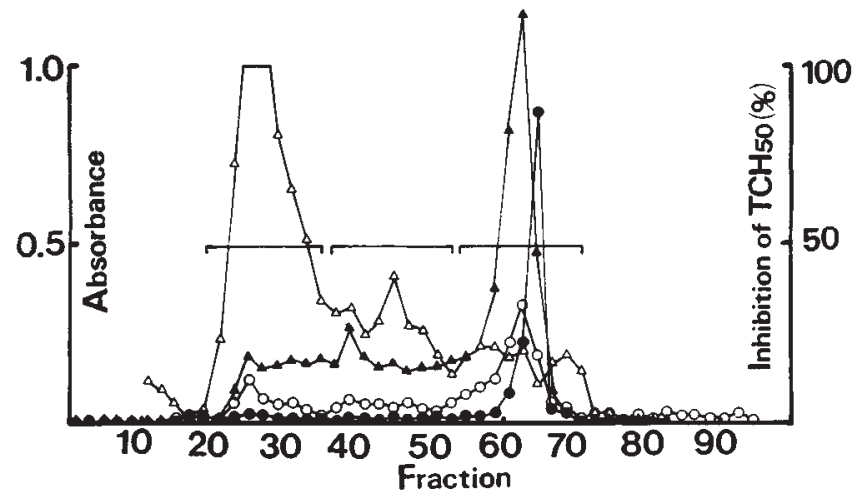

Fig. 4. Gel filtration of AAF-IIb/RCA unbound fraction on Sephadex G-100. The Sephadex G-100 column $(3 \times 87 \mathrm{~cm})$ was equilibrated with $\mathrm{H}_{2} \mathrm{O}$ and elution was performed with $\mathrm{H}_{2} \mathrm{O}$. Carbohydrate, $490 \mathrm{~nm}(\mathrm{O})$; protein, $280 \mathrm{~nm}(\bullet)$; uronic acid, $520 \mathrm{~nm}$ (A); anti-complementary activity $(\triangle)$. turonic acid. When the RCA-unbound fraction was subjected to gel filtration on Sepharose CL-2B by the elution with water, broad carbohydrate fractions were obtained but the active fraction was eluted near in the void volume (Fig. 5). This result

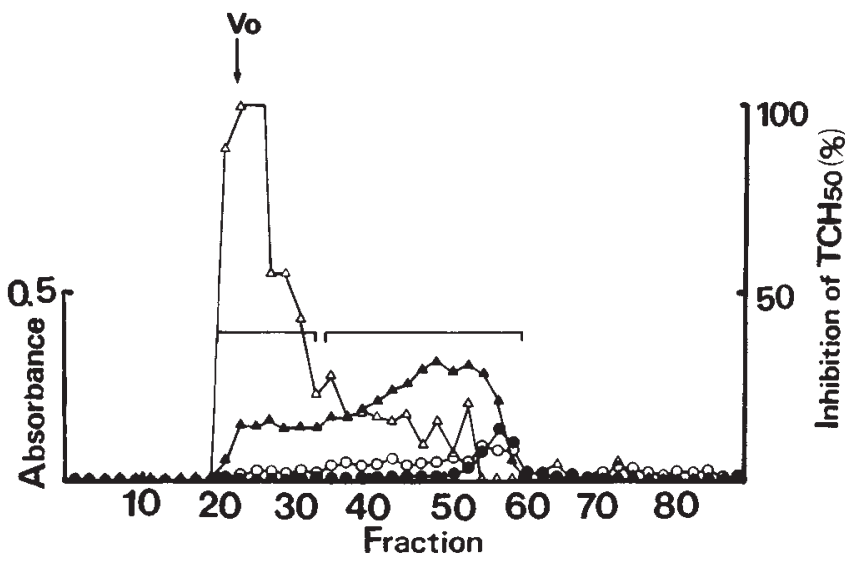

Fig. 5. Gel filtration of AAF-IIb/RCA unbound fraction on Sepharose CL-2B. The Sepharose CL-2B column $(3 \times 90 \mathrm{~cm})$ was equilibrated with distilled $\mathrm{H}_{2} \mathrm{O}$ and elution was performed with $\mathrm{H}_{2} \mathrm{O}$. Symbols expressed same as Fig. 4.

suggests that the active polysaccharide molecules tend to form an aggregate. Therefore the fraction eluted in the void volume by Sephadex G-100 gel filtration was applied on Sepharose CL4B chromatography in the $0.2 \mathrm{M} \mathrm{NaCl}$ solution to determine whether the apparent large molecular weight of the active polysaccharide resulted from aggregation of smaller polysaccharides. Under this condition, small amount of polysaccharide (AAF-IIb-1) voided the column but major polysaccharides (AAF-IIb-2 and 3) were included in the gel (Fig. 6). The colorimetrical assay elution profile of these main polysaccharides (AAF-IIb-2 and 3) showed that there are fractions of relatively enriched in uronosyl residues (AAF-IIb-2) and fractions enriched in neutral sugars (AAF-IIb-3). AAF-IIb-1 and IIb-2 were shown to have the more potent activity than AAF-IIb-3 (Fig. 7). AAF-IIb-2 and IIb-3 gave a single spot on glass-fiber paper electrophoresis in the acetate buffer and borate buffer, respectively.

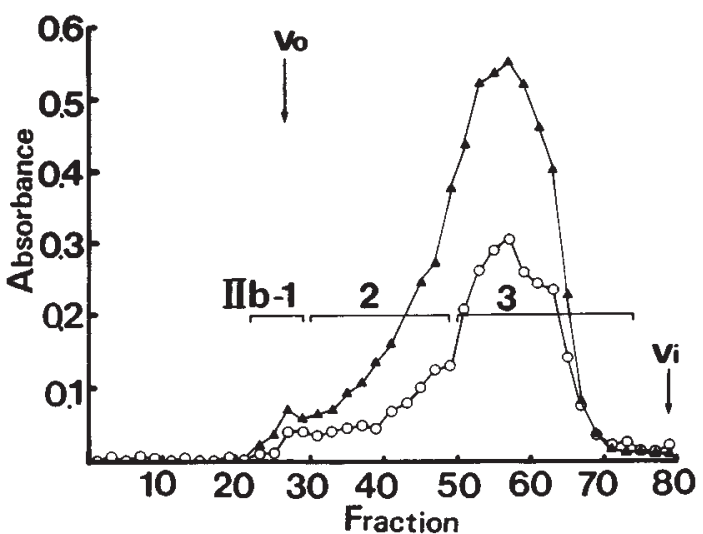

Fig. 6. Gel filtration of Sephadex G-100 void volume fraction from Fig. 4 on Sepharose CL-4B.

The Sepharose CL-4B column $(3 \times 92 \mathrm{~cm})$ was equilibrated with $0.2 \mathrm{M}$ $\mathrm{NaCl}$ and elution was performed with $0.2 \mathrm{M} \mathrm{NaCl}$. Carbohydrate, 490 $\mathrm{nm}(\mathrm{O})$; uronic acid, $520 \mathrm{~nm}(\boldsymbol{\Delta})$. 

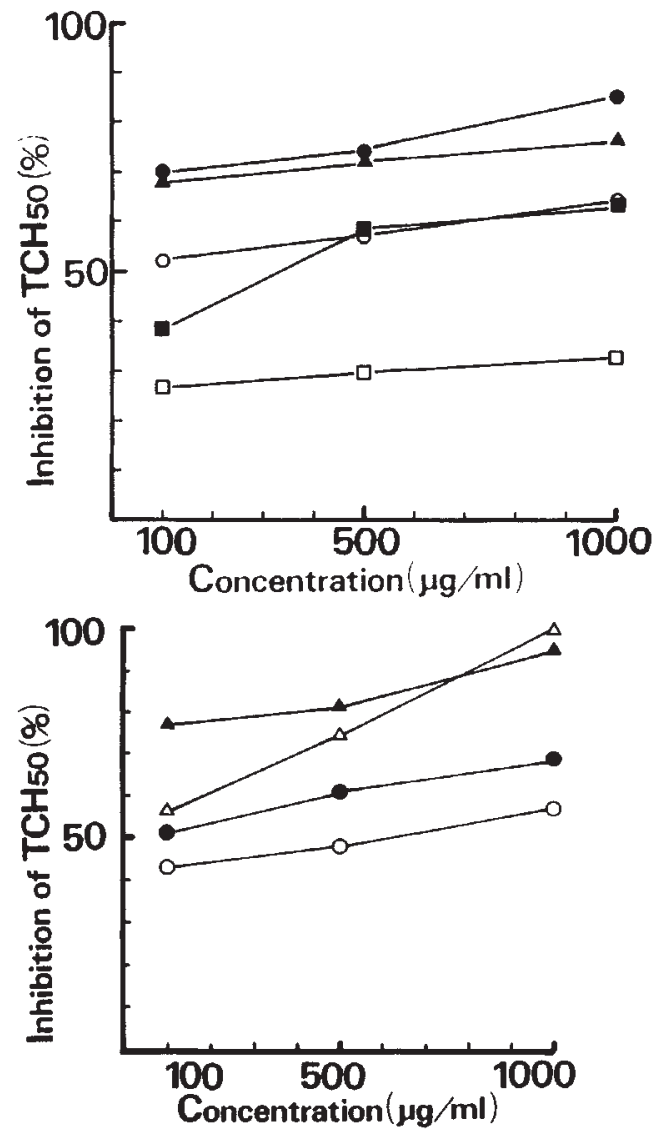

Fig. 7. Anti-complementary activity of purified anti-complementary polysaccharides, AAF-IIb-1, 2 and 3, from Fig. 6 on Sepharose CL-4B. $\triangle$ AAF-IIb-1; AAF-IIb-2; O AAF-IIb-3; $\square$ lentinan; crude ARarabinogalactan from $A$. acutiloba Kitagawa.

Properties of anti-complementary polysaccharides, AAF-IIb-2 and $A A F-I I b-3$

The molecular weights of AAF-IIb- 2 and IIb- 3 were estimated to be 139000 and 31000 by the calibration of gel filtration on Sepharose CL-4B (0.2 M NaCl). AAF-IIb-3 contained a more substantial amount of hexose than AAF-IIb-2, while AAF-IIb2 contained a more substantial amount of uronic acid than AAF-IIb-3 (Table II). AAF-IIb-2 was composed of arabinose, galactose, rhamnose, xylose and a small amount of glucose

Table II. Properties of Anti-complementary Polysaccharides from A. princeps

\begin{tabular}{lcc}
\hline & AAF-IIb-2 & AAF-IIb-3 \\
\hline $\begin{array}{l}\text { Yield (from Sephadex G-100 } \\
\quad \text { void volume) }\end{array}$ & $27 \%$ & $60 \%$ \\
Molecular weight & 139,000 & 31,000 \\
$\quad$ (Sepharose CL-4B in 0.2 M NaCl) & & \\
Total hexose (\%) & 77.5 & 99.0 \\
Total uronic acid (\%) & 59.4 & 49.0 \\
Total protein (\%) & 2.7 & 3.2 \\
\hline Component sugars) & \multicolumn{2}{c}{ (Molar ratios) } \\
Rhamnose & 2.5 & 1.5 \\
Xylose & 2.5 & 1.0 \\
Arabinose & 4.3 & 9.4 \\
Galactose & 3.6 & 7.5 \\
Glucose & 1.0 & 1.0 \\
\hline
\end{tabular}

while AAF-IIb-3 was composed of arabinose, galactose and small amounts of rhamnose, xylose and glucose. The anti-complementary activity of AAF-IIb-3 was almost similar with that of crude arabinogalactan from the root of Angelica acutiloba Kitagawa, but AAF-IIb-2 and IIb-3 showed more potent activity than anti-complementary glucan, lentinan (Fig. 7).

\section{Discussion}

It has been reported that the several immunomodulating activities were found in the crude polysaccharide fractions of the higher plants (15-17), however further purification of the active polysaccharide was not carried out in these studies.

The extract of the leaves of $A$. princeps Pamp was found to contain potent anti-complementary activity, and major anticomplementary polysaccharides from $A$. princeps Pamp have now been purified and are characterized in the present paper. Anti-complementary activity was shown to be present in the crude polysaccharide fraction but not in other fractions. The activity was observed in the neutral and acidic polysaccharide fractions. The acidic major polysaccharide fraction, AAF-IIb, showed potent activity which was significantly reduced after periodate oxidation but not after Pronase treatment. These results indicate that the carbohydrate moiety contributes to the activity. The major anti-complementary polysaccharides from AAF-IIb did not have affinities to RCA and Con-A. This suggests that the significant amounts of galactosyl and glucosyl residues are not present at the non-reducing ends. The anticomplementary polysaccharide from AAF-IIb showed a broad single peak by gel filtration on Sepharose CL-4B, but AAF-IIb contained at least three kinds of anti-complementary polysaccharides which have a different molecular weight. The present results indicate that the major anti-complementary polysaccharides, AAF-IIb-2 and IIb-3, are probably structurally related polymers because both polysaccharides were composed of the same component sugars, but their molar ratios differed from each other. Both polysaccharides contained remarkable amounts of arabinose and galactose, and their molar ratios were almost same value $(1.2: 1.0)$. It was suggested that AAFIIb-2 and IIb-3 may contain similar arabinogalactan moiety. In a previous study it was shown by this research group that an anti-complementary arabinogalactan was isolated from the root of $A$. acutiloba Kitagawa (AR-arabinogalactan IIa) (12, 18). Crude AR-arabinogalactan IIa showed almost similar activity with AAF-IIb-3. It was expected that arabinogalactan moiety may participate in the anit-complementary activty.

Some anti-complementary polysaccharides have already been isolated from bacteria, fungi and plants, but not yet from Chinese crude drugs except $\beta$-1,3-glucan from Poria $\operatorname{cocos}(19)$, Lentinas edodes (19) and Coriolus versicolar (20) or ARarabinogalactan IIa from $A$. acutiloba Kitagawa $(12,18)$. The major anti-complementary polysaccharides from $A$. princeps Pamp showed more potent activity than anti-complementary $\beta$-1,3-glucan from $L$. edodes. AAF-IIb-2 and IIb-3 contained remarkable amounts of galacturonic acid. But uronic acid containing anti-complementary polysaccharide has not yet been reported. Another acidic polysaccharide, pectin from apple or A. acutiloba did not show the significant anti-complementary activity in comparison with AAF-IIb-2 and IIb-3 (unpublished). These facts indicate that the anti-complementary acidic heteroglycan from $A$. princeps Pamp plays important role in the effect of Chinese crude drugs, and is also useful for the study of complement system. 
Further studies of the structural analysis and mode of anticomplementary activity of this unique acidic heteroglycan are now in progress.

\section{Acknowledgements}

We are grateful to Dr. G. Chihara (National Cancer Center, Japan) for a kind gift of lentinan. An equipment fund from Tsumura-Juntendo Co., Ltd., Tokyo, Japan supported a part of this work.

\section{References}

(1) Cyong, J.-C., Otsuka, Y. (1982) Acupuncture of ElectroTherapeutics Res. 7, 173.

(2) Shindoh, M., Cyong, J.-C., Yamada, H., Kiyohara, H., Otsuka, Y. (1983) Proc. symp. Wakan-Yaku 16, 76.

(3) Shindoh, M., Cyong, J.-C. (1982) Abstract of the annual meeting of the Japanese Society for Oriental Medicine, 27.

(4) Dubois, M., Gilles, K. A., Hamilton, J. K., Rebers, P. A., Smith, F. (1956) Anal. Chem. 28, 350.

(5) Blumenkranz, N., Asboe-hansen, G. (1973) Anal. Biochem. 54, 484.

(6) Lowry, O. H., Rosebrough, N. J., Farr, I., Randall, R. J. (1951) J. Biol. Chem. 193, 265.
(7) Trevelyan, W. E., Procter, D. P., Harrison, J. S. (1950) Nature (London) 166, 444.

(8) Hough, L., Jones, J. K. N., Wadman, W. H. (1950) J. Chem. Soc. 1702.

(9) Yamada, H., Kiyohara, H., Otsuka, Y. (1984) Phytochem. 23, 587.

(10) Kennedy, J. F., Rosevear, A. (1973) J. Chem. Soc. Perkin I. 19, 2041.

(11) Dische, Z. (1962) Methods Carbohydr. Chem. 1, 478.

(12) Yamada, H., Kiyohara, H., Cyong, J.-C., Kojima, Y., Kumazawa, Y., Otsuka, Y. (1984) Planta Medica 50, 163.

(13) Adair, W. E., Kornfeld, S. (1974) J. Biol. Chem. 249, 4696.

(14) Thomas, T.-S. L, Steven, S.-L. L. (1980) Eur. J. Biochem. 105, 453.

(15) Belin, M., Hardy, W. G., Perrault, A., Sato, H. (1959) Cancer Res. 19, 1050.

(16) Caldes, G., Prescott, B., Thomas II, C. A., Barker, P. J. (1981) J. Gen. Appl. Microbiol. 27, 157.

(17) Bloksma, N., Schmiermann, P., Reuver, M., Dijk, H., Willers, J. (1982) Planta Medica 46, 221.

(18) Yamada, H., Kiyohara, H., Cyong, J.-C., Otsuka, Y. (1985) Molec. Immun. In press.

(19) Okuda, T., Yoshioka, Y., Ikekawa, T., Chihara, G., Nishioka, K. (1972) Nature (Lond.) New Biol. 238, 59.

(20) Shimura, K., Ito, H., Hibasami, H. (1983) Japan. J. Pharmacol. $33,403$.

\title{
Activation of Anthranilic Acid and $N$-Methylanthranilic Acid by Cell-free Extracts from Ruta graveolens Tissue Cultures
}

\author{
A. Baumert ${ }^{1}$, I. N. Kuzovkina ${ }^{2}$ and D. Gröger ${ }^{1,3}$ \\ Received: September 10, 1984; Accepted: December 1, 1984
}

\begin{abstract}
Cell-free extracts of Ruta graveolens tissue cultures obtained after $\left(\mathrm{NH}_{4}\right)_{2} \mathrm{SO}_{4}$ precipitation and gel filtration on Sephadex G-25 catalyze the ATP-dependent activation of $N$-methylanthranilic acid according to the hydroxamate method. The rate of the enzyme reaction was shown to be linear for about $75 \mathrm{~min}$ and was proportional to the protein concentration up to at least $0.75 \mathrm{mg} / \mathrm{ml}$ of the enzyme preparation. This particular enzyme activity was only detectable in acridone alkaloid producing tissue cultures. Apparently activation of $N$-methylanthranilic acid is the second pathway-specific step in acridone alkaloid biosynthesis. It is not yet clarified if the activation takes place via the corresponding acyl adenylate or via phosphorylation.
\end{abstract}

\footnotetext{
${ }^{1}$ Institut für Biochemie der Pflanzen, Akademie der Wissenschaften der DDR, DDR-4020 Halle (Saale), Weinberg 3

${ }^{2}$ K. A. Timiriazev Institute of Plant Physiology, Academy of Sciences of the USSR, Moscow, USSR

3 Address for correspondence
}

\section{Introduction}

A number of protein amino acids and some other closely related compounds i.e. ornithine, nicotinic acid and anthranilic acid are essential precursors in alkaloid biosynthesis.

Usually amino acids are decarboxylated during the course of the formation of various nitrogen-containing heterocyclic ring systems. In this context anthranilic acid occupies an unique position. In anthranilate-derived alkaloids, the carboxyl group is retained and takes part in the biosynthesis of the different heterocycles. To this particular group of secondary metabolites belong the acridone alkaloids which are exclusively found in some genera of the Rutaceae. It was argued for thermodynamical reasons that "activated" anthranilic acid might react with malonyl-CoA in the course of acridone biosynthesis (1). Anthraniloyl-coenzyme A (CoA) thioester seemed to be the most likely candidate. It is now well established that the $N$-methylation of anthranilic acid catalyzed by a particular $N$-methyltransferase is the first pathway-specific step in acridone biosyn- 